\title{
Analytical Bound State Solutions of the Klein-Fock-Gordon Equation for the Sum of Hulthén and Yukawa Potential within SUSY Quantum Mechanics
}

\author{
A. I. Ahmadov $\mathbb{D},{ }^{1,2}$ S. M. Aslanova $\mathbb{D}^{1},{ }^{1}$ M. Sh. Orujova, ${ }^{3}$ and S. V. Badalov $\mathbb{D}^{4}$ \\ ${ }^{1}$ Department of Theoretical Physics, Baku State University, Z. Khalilov st. 23, AZ-1148, Baku, Azerbaijan \\ ${ }^{2}$ Institute for Physical Problems, Baku State University, Z. Khalilov st. 23, AZ-1148, Baku, Azerbaijan \\ ${ }^{3}$ Azerbaijan State University of Economics, Istiglaliyyat st. 22, AZ-1001, Baku, Azerbaijan \\ ${ }^{4}$ Lehrstuhl für Theoretische Materialphysik, Universität Paderborn, 33095 Paderborn, Germany
}

Correspondence should be addressed to A. I. Ahmadov; ahmadovazar@yahoo.com

Received 17 September 2020; Accepted 1 February 2021; Published 16 February 2021

Academic Editor: Sunny Vagnozzi

Copyright (C) 2021 A. I. Ahmadov et al. This is an open access article distributed under the Creative Commons Attribution License, which permits unrestricted use, distribution, and reproduction in any medium, provided the original work is properly cited. The publication of this article was funded by $\mathrm{SCOAP}^{3}$.

\begin{abstract}
The relativistic wave equations determine the dynamics of quantum fields in the context of quantum field theory. One of the conventional tools for dealing with the relativistic bound state problem is the Klein-Fock-Gordon equation. In this work, using a developed scheme, we present how to surmount the centrifugal part and solve the modified Klein-Fock-Gordon equation for the linear combination of Hulthén and Yukawa potentials. In particular, we show that the relativistic energy eigenvalues and corresponding radial wave functions are obtained from supersymmetric quantum mechanics by applying the shape invariance concept. Here, both scalar potential conditions, which are whether equal and nonequal to vector potential, are considered in the calculation. The energy levels and corresponding normalized eigenfunctions are represented as a recursion relation regarding the Jacobi polynomials for arbitrary $l$ states. Beyond that, a closed form of the normalization constant of the wave functions is found. Furthermore, we state that the energy eigenvalues are quite sensitive with potential parameters for the quantum states. The nonrelativistic and relativistic results obtained within SUSY QM overlap entirely with the results obtained by ordinary quantum mechanics, and it displays that the mathematical implementation of SUSY quantum mechanics is quite perfect.
\end{abstract}

\section{Introduction}

The exactly solvable problems for quantum systems have long been a subject of intense study in many branches of quantum physics. The main aim of an analytical solution of wave equations for this attention is that the wave function contains all the requisite information for the full description of a quantum system [1-6]. In physics, especially the relativistic quantum mechanical applications to particle and nuclear physics, the relativistic wave equations predict particles' reaction at high energies [5-7]. The analytical solution of the Klein-Fock-Gordon (KFG) equation with physical potentials plays a central role in relativistic quantum mechanics since this wave equation perfectly defines the spinless pseudoscalar pions and Higgs boson.
In principle, numerous methods were developed, and they are still successfully implemented in solving the nonrelativistic and relativistic wave equations with some familiar potentials. The Nikiforov-Uvarov method [8], factorization method [9], Laplace transform approach [10], and the path integral method [11] and shifted 1/N expansion approach $[12,13]$ are used for solving radial and azimuthal parts of the wave equations exactly or quasiexactly in $l \neq 0$ for various potentials. Additionally, there are numerous interesting research works about the KFG equation with physical potentials by using different methods in the literature [14-26]. Among them, as an example, in Ref. [22], the s-wave KFG equation with the vector Hulthén type potential was treated by the standard method. As reported by Talukdar et al., the scattering state solutions of the $s$-wave KFG equation with 
the vector and scalar Hulthén potentials were obtained for the irregular and regular boundary conditions [25]. Besides, the supersymmetry method (SUSY) was also proposed for solving the wave equations analytically [27-30]. Nonetheless, Okon et al. reported analytical solutions of the Schrödinger equation for the Hulthén-Yukawa plus inversely quadratic potential. [31] In Ref. [32-39], the scalar potential, which is nonequal and equal to the vector potential, was supposed to get the bound states of the KFG equation for some typical potential from the ordinary quantum mechanics. Furthermore, KFG equation with the ring-shaped potential was investigated by Dong et al. [36]. If the condition where the interaction potential is insufficient to form antiparticleparticle pairs is considered, the KFG and Dirac equations can be utilized for the investigation of zero- and 1/2-spin particles, respectively.

When a particle is in a strong field, the relativistic wave equations should be considered in the quantum system. In any case, it can be corrected quickly for nonrelativistic quantum mechanics. The Hulthén potential is one of the essential short-range potentials in physics, extensively used to describe the continuum and bound states of the interaction systems. It has been applied to several research areas such as nuclear and particle, atomic, chemical, and condensed matter physics, so analyzing relativistic effects for a particle under this potential could become significant, especially for strong coupling. The Hulthén potential is defined as

$$
V_{H}(r)=-\frac{Z e^{2}}{a} \cdot \frac{e^{-r / a}}{1-e^{-r / a}}
$$

where $Z$ and $a$ are the atomic number and the screening parameter, respectively. They determine the range for the Hulthén potential [40]. The Yukawa potential was proposed in 1935 as an operative potential to describe the strong interactions between nucleons [41]. It takes the following form:

$$
V_{Y}(r)=-\frac{A e^{-k r}}{r},
$$

where $A$ describes the strength of the interaction and $1 / k$, its range. Unfortunately, for arbitrary $l$ states $(l \neq 0)$, the KFG equation cannot get an exact solution with these potentials due to the centrifugal term of potentials. The numerous research works reveal the SUSY QM method's power and simplicity in solving wave equations of the central and noncentral potentials for arbitrary $l$ states [42-49].

In principle, the radial function nature at the origin was investigated particularly for singular potentials by Khelashvili et al. $[50,51]$. While the Laplace operator is portrayed in spherical coordinates, the radial wave equation's exact derivation demonstrates the perspective of a delta function term. Thus, the delta function term of the Laplace operator yields an essential contribution to the energy level. Although the various research attempts have provided satisfactory bound state energies using Hulthén and Yukawa potentials separately [1, 52-57], we first considered these potentials under the linear combination form. [58] It is also worth mentioning that this potential can be used in nuclear physics to investi- gate the interaction between the deformed pair of the nucleus and spin-orbit coupling for the particle motion in the potential fields. Another fascinating perspective of this potential can be used as a mathematical model in the description of vibrations on the hadronic system's side, and it can constitute a convenient model for other physical situations. The investigation of the relativistic bound states in the arbitrary $l$ -wave KFG equation with the linear combination of Hulthén and Yukawa potentials is quite interesting, and it can provide the deeper and accurate appreciations of the physical properties of the wave functions and energies in the continuum and bound states of the interacting systems. Inspired by all developments and works, in this paper, we present the solution of the relativistic radial KFG equation for the linear combination of Hulthén and Yukawa potentials, defined as

$$
V(r)=-\frac{V_{0} e^{-2 \delta r}}{1-e^{-2 \delta r}}-\frac{A e^{-\delta r}}{r},
$$

where $V_{0}=2 \delta Z e^{2}$ and $\delta$ is the screening parameter.

To study the system, we use an improved scheme to overcome the centrifugal term and the SUSY quantum mechanics $[59,60]$. Despite our previous research effort on this potential [58], the investigation of this potential still needs to be clarified in detail. Accordingly, the main goal is to solve the KFG equation for the linear combination of Hulthén and Yukawa potentials by considering two cases, i.e., the scalar potential which is equal and unequal to vector potential by using SUSY QM. Thereby, the energy eigenvalues and corresponding radial wave functions are found for any $l$ orbital angular momentum case. Then, we compare the obtained results with the results obtained by the NU method in ordinary quantum mechanics to present the legitimacy and feasibility of this SUSY QM method. The remainder of the paper is structured as follows. In Section 2, we introduce the analytical solution of the radial KFG equation for the linear combination of Hulthén and Yukawa potentials from SUSY quantum mechanics. Next, the analysis of the results is presented in Section 3. Finally, Section 4 contains the conclusions.

\section{Bound State Solution of the Radial Klein- Fock-Gordon Equation}

2.1. Implementation SUSY Quantum Mechanics. Two different types of potential can be introduced into the KFG equation, which contains two objects: (i) the four-vector linear momentum operator and (ii) the scalar rest mass. Hence, the first one is a vector potential $V$, which is introduced via minimal coupling, and the second one is a scalar potential $S$ , which is introduced via scalar coupling [5]. At this moment, they allow one to introduce two types of potential coupling: the vector potential $V$ and the space-time scalar potential $S$. The natural units $(\hbar=c=1)$ are set throughout this study. In the spherical coordinates systems, the KFG equation with vector potential $V(r, \theta)$ and scalar potential $S(r, \theta)$ has the form

$$
\left[-\nabla^{2}+(M+S(r))^{2}\right] \psi(r, \theta, \phi)=[E-V(r)]^{2} \psi(r, \theta, \phi),
$$


where $E$ is the relativistic energy and $M$ denotes the rest mass of the system's scalar particle. For the separation of the angular and radial parts of the wave function, in the stationary KFG equation with the linear combination of Hulthén and Yukawa potentials, the wave function should utilize the following wave function:

$$
\psi(r, \theta, \phi)=\frac{\chi(r)}{r} \Theta(\theta) e^{i m \phi}, m=0, \pm 1, \pm 2, \pm 3 \cdots
$$

and substituting this into Equation (4), the radial KFG equation is defined in the following form:

$\chi^{\prime \prime}(r)+\left[\left(E^{2}-M^{2}\right)-2(M \cdot S(r)+E \cdot V(r))+\left(V^{2}(r)-S^{2}(r)\right)-\frac{l(l+1)}{r^{2}}\right] \chi(r)=0$.

As it is known that the KFG equation with this potential can be solved exactly using a suitable approximation scheme to surmount the centrifugal term. To solve Equation (6) for $l \neq 0$, we ought to approximate the centrifugal term of the Yukawa potential in this system. As a result of this, while $\delta$ $r<<1$, the improved approximation scheme [61-65] must be used as

$$
\begin{gathered}
\frac{1}{r} \approx \frac{2 \delta e^{-\delta r}}{1-e^{-2 \delta r}}, \\
\frac{1}{r^{2}} \approx \frac{4 \delta^{2} e^{-2 \delta r}}{\left(1-e^{-2 \delta r}\right)^{2}} .
\end{gathered}
$$

Next, the vector and scalar potential forms for the general Hulthén and Yukawa potentials can be considered in the following forms:

$$
\begin{aligned}
& V_{H}(r)=-\frac{V_{0} e^{-2 \delta r}}{1-e^{-2 \delta r}}, S_{H}(r)=-\frac{S_{0} e^{-2 \delta r}}{1-e^{-2 \delta r}}, \\
& V_{Y}(r)=-\frac{V_{0}{ }^{\prime} e^{-2 \delta r}}{1-e^{-2 \delta r}}, S_{Y}(r)=-\frac{S_{0}{ }^{\prime} e^{-2 \delta r}}{1-e^{-2 \delta r}} .
\end{aligned}
$$

Then, Equation (6) becomes

$$
\begin{aligned}
\chi^{\prime \prime}(r) & +\left[\left(E^{2}-M^{2}\right)+2\left(\frac{M\left(S_{0}+S_{0}{ }^{\prime}\right)+E\left(V_{0}+V_{0}{ }^{\prime}\right)}{1-e^{-2 \delta r}}\right) e^{-2 \delta r}\right. \\
& \left.+\left(\frac{\left(V_{0}+V_{0}{ }^{\prime}\right)^{2}-\left(S_{0}+S_{0}{ }^{\prime}\right)^{2}}{\left(1-e^{-2 \delta r}\right)^{2}}\right) e^{-4 \delta r}-\frac{4 l(l+1) \delta^{2} e^{-2 \delta r}}{\left(1-e^{-2 \delta r}\right)^{2}}\right] \chi(r)=0 .
\end{aligned}
$$

Thereby, the effective potential of the Hulthén and
Yukawa potential linear combination has the following form:

$$
V_{\mathrm{eff}}(r)=-\frac{4 \delta^{2}\left(\alpha^{2}+\beta^{2}\right) e^{-2 \delta r}}{1-e^{-2 \delta r}}-\frac{4 \delta^{2}\left(\gamma^{2}-\rho^{2}\right) e^{-4 \delta r}}{\left(1-e^{-2 \delta r}\right)^{2}}+\frac{4 l(l+1) \delta^{2} e^{-2 \delta r}}{\left(1-e^{-2 \delta r}\right)^{2}},
$$

where

$$
\varepsilon=\frac{\sqrt{M^{2}-E^{2}}}{2 \delta}>0
$$

$$
\begin{gathered}
\alpha=\frac{\sqrt{2 E V_{0}+2 M S_{0}}}{2 \delta}>0, \\
\beta=\frac{\sqrt{2 E V_{0}^{\prime}+2 M S_{0}^{\prime}}}{2 \delta}>0, \\
\gamma=\frac{V_{0}+V_{0}^{\prime}}{2 \delta}>0, \\
\rho=\frac{S_{0}+S_{0}^{\prime}}{2 \delta}>0 .
\end{gathered}
$$

For investigation in detail, the nonrelativistic limit of the formula must be studied for the energy level. When $V(r)=$ $S(r)$, Equation (4) reduces to a Schrödinger equation for the potential $2 V(r)$. Based on supersymmetric quantum mechanics, the eigenfunction of ground state $\chi_{0}(r)$ in Equation (6) should be in the following form:

$$
\chi_{0}(r)=N \exp \left(-\int W(r) d r\right)
$$

where $N$ and $W(r)$ are normalised constant and superpotential, respectively. The connection between the supersymmetric partner potentials $V_{-}(r)$ and $V_{+}(r)$ of the superpotential $W(r)$ is as follows $[27,28]$ :

$$
\begin{aligned}
& V_{-}(r)=W^{2}(r)-W^{\prime}(r), \\
& V_{+}(r)=W^{2}(r)+W^{\prime}(r) .
\end{aligned}
$$

The particular solution of the Riccati equation (Equation (13)) must be in the following form:

$$
W(r)=-\left(F+\frac{G e^{-2 \delta r}}{1-e^{-2 \delta r}}\right)
$$

where $G$ and $F$ are unknown constants. Having inserted Equation (14) into Equation (13) and taking into account that $V_{-}(r)=V_{\text {eff }}(r)-\left(E^{2}-M^{2}\right)$, we obtain 


$$
\begin{aligned}
F^{2}+ & \frac{2 F G e^{-2 \delta r}}{1-e^{-2 \delta r}}+\frac{G^{2} e^{-4 \delta r}}{\left(1-e^{-2 \delta r}\right)^{2}}-\frac{2 \delta G e^{-2 \delta r}}{1-e^{-2 \delta r}}-\frac{2 \delta G e^{-4 \delta r}}{\left(1-e^{-2 \delta r}\right)^{2}} \\
= & -\frac{4 \delta^{2}\left(\alpha^{2}+\beta^{2}\right) e^{-2 \delta r}}{1-e^{-2 \delta r}}-\frac{4 \delta^{2}\left(\gamma^{2}-\rho^{2}\right) e^{-4 \delta r}}{\left(1-e^{-2 \delta r}\right)^{2}} \\
& +\frac{4 l(l+1) \delta^{2} e^{-2 \delta r}}{\left(1-e^{-2 \delta r}\right)^{2}}-\left(E^{2}-M^{2}\right) .
\end{aligned}
$$

After small simplification, it can be rewritten as

$$
\begin{aligned}
& F^{2}+\frac{(2 F G-2 \delta G) e^{-2 \delta r}}{1-e^{-2 \delta r}}+\frac{\left(G^{2}-2 \delta G\right) e^{-4 \delta r}}{\left(1-e^{-2 \delta r}\right)^{2}} \\
& =4 \delta^{2} \varepsilon^{2}-\frac{4 \delta^{2}\left(\alpha^{2}+\beta^{2}\right) e^{-2 \delta r}}{1-e^{-2 \delta r}}-\frac{4 \delta^{2}\left(\gamma^{2}-\rho^{2}\right) e^{-4 \delta r}}{\left(1-e^{-2 \delta r}\right)^{2}} \\
& \quad+4 l(l+1) \delta^{2}\left[\frac{e^{-2 \delta r}}{1-e^{-2 \delta r}}+\frac{e^{-4 \delta r}}{\left(1-e^{-2 \delta r}\right)^{2}}\right]
\end{aligned}
$$

From comparison of compatible quantities in the left and right sides of the equation (Equation (16)), we find the following relations for $G$ and $F$ constants:

$$
\begin{gathered}
F^{2}=4 \delta^{2} \varepsilon^{2}, \\
2 F G-2 \delta G=4 \delta^{2} l(l+1)-4 \delta^{2}\left(\alpha^{2}+\beta^{2}\right), \\
G^{2}-2 \delta G=-4 \delta^{2}\left(\gamma^{2}-\rho^{2}\right)+4 \delta^{2} l(l+1) .
\end{gathered}
$$

Considering extremity conditions for wave functions, we obtain $G>0$ and $F<0$. Solving Equation (19) yields

$$
G=\delta \pm 2 \delta \sqrt{\left(l+\frac{1}{2}\right)^{2}-\gamma^{2}+\rho^{2}}
$$

and considering $G>0$ from Equations (18) and (19), we find that

$$
F=\frac{G}{2}-\frac{2 \delta^{2}\left(\alpha^{2}+\beta^{2}-\gamma^{2}+\rho^{2}\right)}{G} .
$$

From Equation (17) and Equation (21), we find that

$\varepsilon^{2}=\frac{1}{4 \delta^{2}}\left[\frac{\delta+2 \delta \sqrt{(l+1 / 2)^{2}-\gamma^{2}+\rho^{2}}}{2}-\frac{2 \delta\left(\alpha^{2}+\beta^{2}-\gamma^{2}+\rho^{2}\right)}{1+2 \sqrt{(l+1 / 2)^{2}-\gamma^{2}+\rho^{2}}}\right]^{2}$.

After inserting (22) into (11) for the definitions of the energy eigenvalue of ground state for general case $V(r)$ $\neq S(r)$, we obtain the following energy level equation:

$$
M^{2}-E_{0}^{2}=\left[\frac{\delta+2 \delta \sqrt{(l+(1 / 2))^{2}-\gamma^{2}+\rho^{2}}}{2}-\frac{2 \delta\left(\alpha^{2}+\beta^{2}-\gamma^{2}+\rho^{2}\right)}{1+2 \sqrt{(l+(1 / 2))^{2}-\gamma^{2}+\rho^{2}}}\right]^{2} .
$$

When $r \rightarrow \infty$, the chosen superpotential $W(r) \rightarrow-F$. Inserting Equation (14) into Equation (13), the supersymmetric partner potentials $V_{-}(r)$ and $V_{+}(r)$ can be found in the following forms:

$$
\begin{aligned}
& V_{-}(r)=F^{2}+\frac{(2 F G-2 \delta G) e^{-2 \delta r}}{1-e^{-2 \delta r}}+\frac{\left(G^{2}-2 \delta G\right) e^{-4 \delta r}}{\left(1-e^{-2 \delta r}\right)^{2}}, \\
& V_{+}(r)=F^{2}+\frac{(2 F G+2 \delta G) e^{-2 \delta r}}{1-e^{-2 \delta r}}+\frac{\left(G^{2}+2 \delta G\right) e^{-4 \delta r}}{\left(1-e^{-2 \delta r}\right)^{2}} .
\end{aligned}
$$

By using the superpotential $W(r)$ from Equation (14), we can find $\chi_{0}(r)$ radial eigenfunction of ground state in the following form:

$$
\chi_{0}(r)=N e^{F r}\left(1-e^{-2 \delta r}\right)^{G / 2 \delta}
$$

where $r \rightarrow 0 ; \chi_{0}(r) \rightarrow 0, G>0$, and $r \rightarrow \infty ; \chi_{0}(r) \rightarrow 0, F$ $<0$. Two partner potentials $V_{-}(r)$ and $V_{+}(r)$ which differ from each other with additive constants and have the same functional form are called the invariant potentials $[59,60]$. Hence, for the partner potentials $V_{-}(r)$ and $V_{+}$ $(r)$ given with Equations (13) and (14), the invariant forms are defined as

$$
\begin{aligned}
R\left(G_{1}\right)= & V_{+}(G, r)-V_{-}\left(G_{1}, r\right)=F^{2}-F_{1}^{2}=\left[\frac{G}{2}-\frac{2 \delta^{2}\left(\alpha^{2}+\beta^{2}-\gamma^{2}+\rho^{2}\right)}{G}\right]^{2} \\
& -\left[\frac{G+2 \delta}{2}-\frac{2 \delta^{2}\left(\alpha^{2}+\beta^{2}-\gamma^{2}+\rho^{2}\right)}{G+2 \delta}\right]^{2}
\end{aligned}
$$

$$
\begin{aligned}
R\left(G_{i}\right)= & V_{+}[G+(i-1) 2 \delta, r]-V_{-}[G+i 2 \delta, r] \\
= & \left(\frac{G+(i-1) \cdot 2 \delta}{2}-\frac{2\left(\alpha^{2}+\beta^{2}-\gamma^{2}+\rho^{2}\right) \delta^{2}}{G+(i-1) \cdot 2 \delta}\right)^{2} \\
& -\left(\frac{G+i \cdot 2 \delta}{2}-\frac{2\left(\alpha^{2}+\beta^{2}-\gamma^{2}+\rho^{2}\right) \delta^{2}}{G+i \cdot 2 \delta}\right)^{2} .
\end{aligned}
$$

where the reminder $R\left(G_{i}\right)$ is independent of $r$. If we keep going this procedure and make the following substitution $G_{n_{r}}=G_{n_{r}-1}+2 \delta=G+2 n_{r} \delta$, the whole discrete level of Hamiltonian $H_{-}(G)$ can be written as

$$
E_{n_{r}}^{2}=E_{0}^{2}+\sum_{i=1}^{n} R\left(G_{i}\right)
$$

and we obtain the following form: 


$$
E_{n_{r} l}^{2}=M^{2}-\left(\frac{G+2 \delta n_{r}}{2}-\frac{2 \delta^{2}\left(\alpha^{2}+\beta^{2}-\gamma^{2}+\rho^{2}\right)}{G+2 \delta n_{r}}\right)^{2}
$$

In the following, we obtain the energy level equation in ordinary quantum mechanics

$$
M^{2}-E_{n_{r} l}^{2}=\left[\frac{\alpha^{2}+\beta^{2}-(l+(1 / 2))^{2}-\left(n_{r}+(1 / 2)\right)^{2}-2\left(n_{r}+(1 / 2)\right) \sqrt{(l+(1 / 2))^{2}-\gamma^{2}+\rho^{2}}}{n_{r}+(1 / 2)+\sqrt{(l+(1 / 2))^{2}-\gamma^{2}+\rho^{2}}} \cdot \delta\right]^{2}
$$

As seen from Equation (30), it is in a perfect agreement with the result obtained in Equation (27) of Ref. [49]. If we consider Equation (11) into Equation (30) and do some simple algebraic derivation, we can obtain the energy eigenvalue equation in the simplest form

$$
\begin{aligned}
M^{2}-E_{n_{r} l}^{2}= & {\left[\delta\left(n_{r}+\frac{1}{2}+\sqrt{\left(l+\frac{1}{2}\right)^{2}-\gamma^{2}+\rho^{2}}\right)\right.} \\
& \left.-\frac{\gamma E_{n_{r} l}+\rho M+\delta\left(\rho^{2}-\gamma^{2}\right)}{n_{r}+(1 / 2)+\sqrt{(l+(1 / 2))^{2}-\gamma^{2}+\rho^{2}}}\right]^{2}
\end{aligned}
$$

with $\alpha^{2}+\beta^{2}=\left(\gamma E_{n_{l} l}+\rho M\right) / \delta$.

Based on the SUSY QM method and knowing the ground state eigenvalues $E_{0}$ and eigenfunctions $\chi_{0}$, all energy eigenvalues $E_{n_{r} l}$ and eigenfunctions $\chi_{n_{r} l}$ can be easily obtained. Briefly, using the following equation:

$$
\chi_{n_{r}}\left(r, a_{0}\right)=A^{+}\left(r, a_{0}\right) \chi_{n_{r}-1}\left(r, a_{1}\right),
$$

$\chi_{n_{r} l}$ can be easily obtained in terms of the ground state wave functions. The superpotential $W(r)$ depends on two parameters $a_{0}=(F, G)$ and the first partner potential has like that parameter $a_{1}=\left(F_{1}, G_{1}\right)$. Hence, Equation (32) will be in the following form:

$$
\chi_{n_{r}}\left(r, a_{0}\right)=\left(-\frac{d}{d r}-F-\frac{G e^{-2 \delta r}}{1-e^{-2 \delta r}}\right) \chi_{n_{r}-1}\left(r, a_{1}\right)
$$

We define a new variable $s=e^{-2 \delta r} \in[0,1]$ and factoring out the ground state wavefunction

$$
\chi_{n_{r}}\left(s, a_{0}\right)=\chi_{0}\left(s, a_{0}\right) R_{n_{r}}\left(s, a_{0}\right)
$$

Substituting into Equation (33) and using the ground state wavefunction (Equation (25)), we get

$R_{n_{r}}(s ; \varepsilon, K)=s(1-s) \frac{d}{d s} R_{n_{r}-1}(s ; \varepsilon, K+1)+[2 \varepsilon-(2 \varepsilon+2 K+1)] R_{n_{r}-1}(s ; \varepsilon, K+1)$.
Based on comparison, it with the recursion relation in Ref [66]:

$$
\begin{aligned}
P_{n_{r}}^{(\alpha, \beta)}(1-2 s)= & s(1-s) \frac{d}{d s} P_{n_{r}-1}^{(\alpha+1, \beta+1)}(1-2 s) \\
& +[\alpha+1-(\alpha+\beta+2) s)] P_{n_{r}-1}^{(\alpha+1, \beta+1)}(1-2 s),
\end{aligned}
$$

it is seen that $R_{n_{r}}\left(s, a_{0}\right)$ is proportional to the Jacobi polynomial $P_{n_{r}}^{(2 \varepsilon, 2 K-1)}(1-2 s)$. Thus, the normalized eigenfunction for this potential is taken in the following form:

$$
\chi_{n_{r} l}(s)=C_{n_{r}} s^{\varepsilon}(1-s)^{K} P_{n_{r}}^{(2 \varepsilon, 2 K-1)}(1-2 s),
$$

$$
\chi_{n_{r} l}(s)=C_{n_{r}} s^{\varepsilon}(1-s)^{K} \frac{\Gamma\left(n_{r}+2 \varepsilon+1\right)}{n_{r} ! \Gamma(2 \varepsilon+1)} \cdot{ }_{2} F_{1}\left(-n_{r}, 2 \varepsilon+2 K+n_{r}, 1+2 \varepsilon ; s\right),
$$

where $K=1 / 2+\sqrt{(l+(1 / 2))^{2}-\gamma^{2}-\rho^{2}}$. The normalization constant $C_{n_{r} l}$ can be found by using the normalization condition

$$
\int_{0}^{\infty}|R(r)|^{2} r^{2} d r=\int_{0}^{\infty}|\chi(r)|^{2} d r=\frac{1}{2 \delta} \int_{0}^{1} \frac{1}{s}|\chi(s)|^{2} d s=1,
$$

by utilizing the following integral formula in Ref. [66]:

$$
\begin{gathered}
\int_{0}^{1}(1-z)^{2(\delta+1)} z^{2 \lambda-1}{ }_{2} F_{1}\left(-n_{r}, 2(\delta+\lambda+1)+n_{r}, 2 \lambda+1 ; z\right)^{2} d z \\
\quad=\frac{\left(n_{r}+\delta+1\right) n_{r} ! \Gamma\left(n_{r}+2 \delta+2\right) \Gamma(2 \lambda) \Gamma(2 \lambda+1)}{\left(n_{r}+\delta+\lambda+1\right) \Gamma\left(n_{r}+2 \lambda+1\right) \Gamma\left(2(\delta+\lambda+1)+n_{r}\right)},
\end{gathered}
$$

where $\lambda>0$ and $\delta>-3 / 2$. After making simple calculations, we arrive at the following expression for the normalization constant:

$$
C_{n_{r} l}=\sqrt{\frac{2 \delta n_{r} !\left(n_{r}+K+\varepsilon\right) \Gamma(2 \varepsilon+1) \Gamma\left(n_{r}+2 \varepsilon+2 K\right)}{\left(n_{r}+K\right) \Gamma(2 \varepsilon) \Gamma\left(n_{r}+2 K\right) \Gamma\left(n_{r}+2 \varepsilon+1\right)}} .
$$




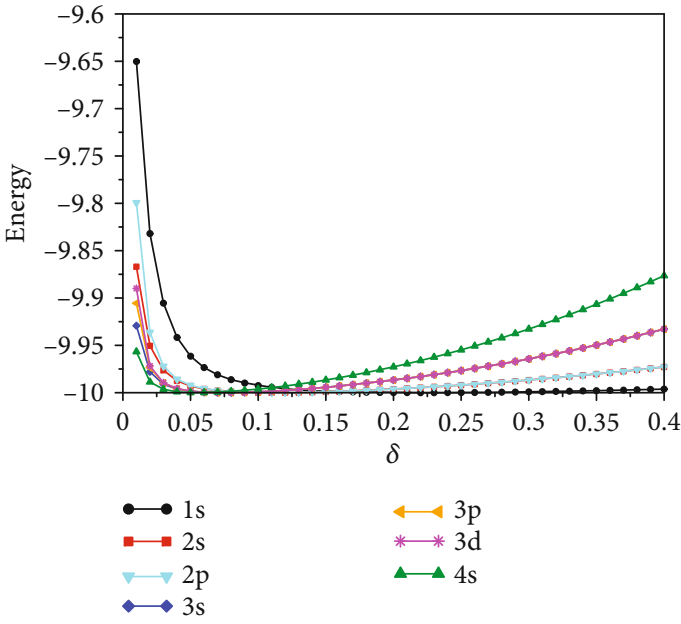

(a)

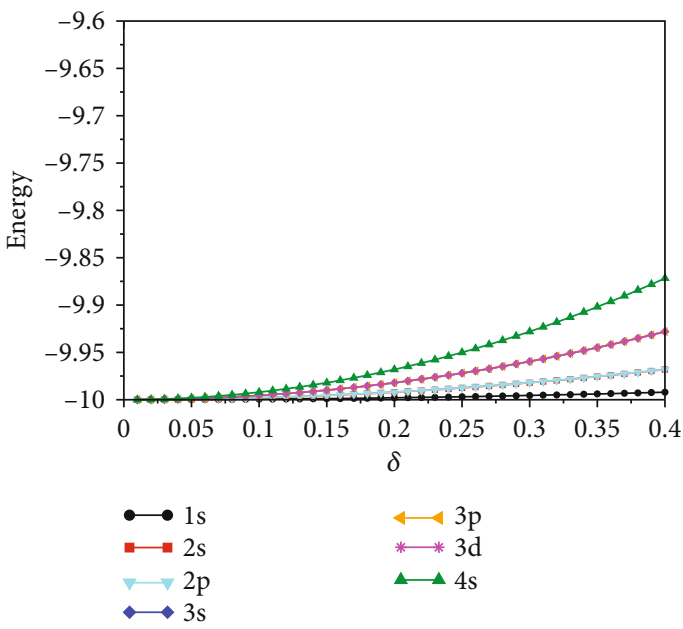

(c)

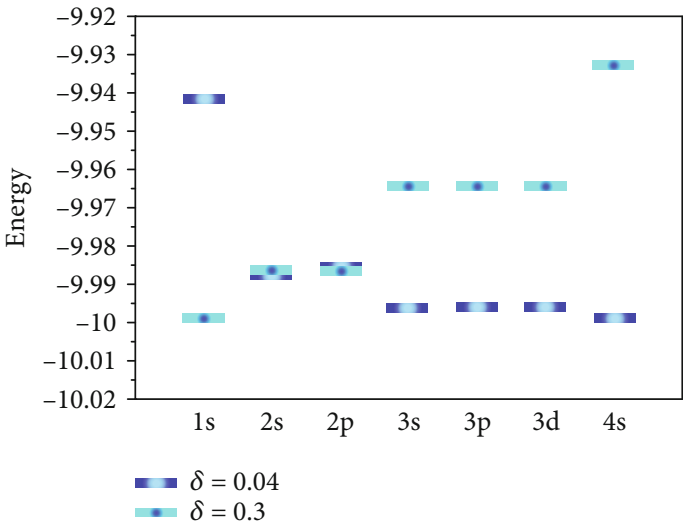

(b)

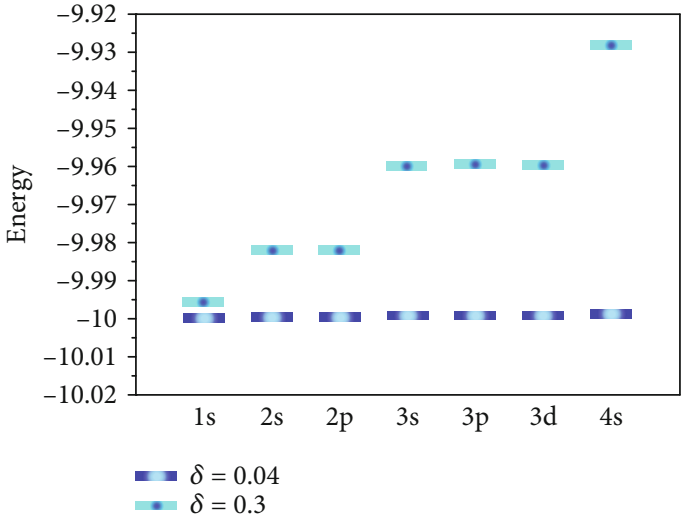

(d)

Figure 1: (Color online). The variation of energy level as a function of screening parameter $\delta$ for quantum states in (a, b) the parameters $M=10, V_{0}=0.01, V_{0}^{\prime}=0.05, S_{0}=0.025$, and $S_{0}^{\prime}=0.035$ and (c, d) the parameters $M=10, V_{0}=0.02, V^{\prime}{ }_{0}=0.06, S_{0}=0.035$, and $S_{0}^{\prime}=$ 0.045 .

\section{Results and Discussion}

In this section, we present the numerical evaluation for the bound state solutions of the $l$-wave KFG equation with the vector and scalar form of the linear combination of Hulthén and Yukawa potentials. To study the property of the energy levels regarding potential parameters in some quantum states, (see Figure 1) we take $M=10, V_{0}=0.01, V_{0}^{\prime}=0.05$, $S_{0}=0.025$, and $S_{0}^{\prime}=0.035$ and $M=10, V_{0}=0.02, V_{0}^{\prime}=$ $0.06, S_{0}=0.035$, and $S_{0}^{\prime}=0.045$. The little difference $(\sim$ 0.01 ) in model potential parameters $V_{0}, V_{0}^{\prime}, S_{0}$, and $S_{0}^{\prime}$ is quite sufficient, in order to see the energy level of quantum states displaye completely different behavior. In Figures 1(a) and 1(b), the energy levels $E$ of quantum states first are decreasing until some of small $\delta$ values $(\sim 0.075-$ 0.1 ), then the energy levels $E$ increase in the $\delta>0.1$. In Figures $1(\mathrm{c})$ and $1(\mathrm{~d})$, the energy levels $E$ of quantum states have very little variation for an interval of $\delta \in[0,0.1]$, and it causes the degenerate of all quantum states, then the energy levels $E$ of quantum states continue to gradually increase with increments of $\delta$. These behaviors are better recognized in higher quantum states.

Behind that of these results, we can investigate some special cases.

(i) In case $S_{0}=V_{0}$, and $S_{0}^{\prime}=V_{0}^{\prime}$, namely, $\gamma=\rho$, we obtain

$$
M^{2}-E_{n_{r} l}^{2}=\left[\delta\left(n_{r}+l+1\right)-\frac{\gamma\left(E_{n_{r} l}+M\right)}{n_{r}+l+1}\right]^{2}
$$

where $\gamma=\left(V_{0}+V_{0}^{\prime}\right) / 2 \delta$

(ii) If $V_{0}^{\prime}=0, S_{0}^{\prime}=0$, we obtain the energy level equation for Hulthén potential case 


$$
\begin{aligned}
M^{2}-E_{n_{r} l}^{2}= & {\left[\delta\left(n_{r}+\frac{1}{2}+\sqrt{\left(l+\frac{1}{2}\right)^{2}-\gamma^{\prime 2}+\rho^{\prime 2}}\right)\right.} \\
& \left.-\frac{\gamma^{\prime} E_{n_{r} l}+\rho^{\prime} M+\delta\left(\rho^{\prime 2}-\gamma^{\prime 2}\right)}{n_{r}+1 / 2+\sqrt{(l+(1 / 2))^{2}-\gamma^{\prime 2}+\rho^{\prime 2}}}\right]^{2},
\end{aligned}
$$

where $\gamma^{\prime}=V_{0} / 2 \delta$ and $\rho^{\prime}=S_{0} / 2 \delta$. This result is in good agreement with the expression obtained in Equation (A.1) of Ref [58]

(iii) In case $V_{0}=0, S_{0}=0$, but $V_{0}^{\prime} \neq S_{0}^{\prime}$, we obtain the energy level equation for Yukawa potential, which is defined as the following form:

$$
\begin{aligned}
M^{2}-E_{n_{r} l}^{2}= & {\left[\delta\left(n_{r}+\frac{1}{2}+\sqrt{\left(l+\frac{1}{2}\right)^{2}-\gamma^{\prime \prime} 2+\rho^{\prime \prime} 2}\right)\right.} \\
& \left.-\frac{\gamma^{\prime \prime} E_{n_{r} l}+\rho^{\prime \prime} M+\delta\left(\rho^{\prime \prime} 2-\gamma^{\prime \prime} 2\right)}{n_{r}+(1 / 2)+\sqrt{(l+(1 / 2))^{2}-\gamma^{\prime \prime} 2+\rho^{\prime \prime} 2}}\right]^{2},
\end{aligned}
$$

where $\gamma^{\prime \prime}=V^{\prime}{ }_{0} / 2 \delta$, and $\rho^{\prime \prime}=S_{0}^{\prime} / 2 \delta$. This result is in good agreement with the expression obtained in Equation (A.3) of Ref [58]. Furthermore, this result is also the same with the expression for the constant mass case obtained in Ref [67]. One can easily see this by setting $q=1$ and $\alpha \rightarrow \delta$ in Equation (39) of Ref [67]

(iv) Also, if $V_{0}=-S_{0}$, and $V_{0}^{\prime}=-S_{0}^{\prime}$, namely, $\gamma=-\rho$

$$
M^{2}-E_{n_{r} l}^{2}=\left[\delta\left(n_{r}+l+1\right)-\frac{\gamma\left(E_{n_{r} l}-M\right)}{n_{r}+l+1}\right]^{2},
$$

where $\gamma=\left(V_{0}+V_{0}^{\prime}\right) / 2 \delta$

(v) If $\delta \rightarrow 0$ and $S_{0}=V_{0}=2 \delta Z e^{2}$ or $\gamma^{\prime}=\rho^{\prime}=V_{0} / 2 \delta=Z$ $e^{2}$ in Equation (31), the potential reduces to Coulomb potential, $V_{c}(r)=-Z e^{2} / r$, and the corresponding energy spectrum is obtained as

$$
E_{n_{r} l}=\frac{\left(n_{r}+l+1\right)^{2}-Z^{2} e^{4}}{\left(n_{r}+l+1\right)^{2}+Z^{2} e^{4}} M,
$$

and this result is the same with Equation (A.2) of Ref [67]

(vi) If we take $l=0$ (the $s$-wave case), the centrifugal term in Equation (9) disappears because $4 l(l+1) \delta^{2} e^{-2 \delta r} /$ $\left(1-e^{-2 \delta r}\right)^{2}=0$ and the equation turns to the $s$ -wave KFG equation. By setting $l=0$ in Equation (31), its energy spectrum equation is the following form:

$$
M^{2}-E_{n_{r} l}^{2}=\left[\delta\left(n_{r}+\frac{1}{2}+\sqrt{\frac{1}{4}-\gamma^{2}+\rho^{2}}\right)-\frac{\gamma E_{n_{r} l}+\rho M+\delta\left(\rho^{2}-\gamma^{2}\right)}{n_{r}+(1 / 2)+\sqrt{(1 / 4)-\gamma^{2}+\rho^{2}}}\right]^{2}
$$

(vii) If we take $S_{0}=V_{0}$ and $S_{0}^{\prime}=V_{0}^{\prime}$, and based on the following transformations: $E_{n_{r} l}-M \rightarrow E_{n_{r} l}^{N R}, E_{n_{r} l}+M$ $\rightarrow 2 M, V_{0} \rightarrow V_{0} / 2$, and $V_{0}^{\prime} \rightarrow V_{0}^{\prime} / 2$, we obtain the energy level equation of Equation (31) for the nonelativistic case. Briefly, because of the following relation:

$$
\begin{gathered}
M^{2}-E_{n_{r} l}^{2}=\left(M-E_{n_{r} l}\right)\left(M+E_{n_{r} l}\right)=-E_{n_{r} l}^{N R} \cdot 2 M, \\
\gamma=\rho=\frac{V_{0}+V_{0}^{\prime}}{4 \delta}=\frac{V_{0}+2 \delta A}{4 \delta}, \\
\gamma\left(E_{n_{r} l}+M\right)=2 M \gamma=\frac{M\left(V_{0}+2 \delta A\right)}{2 \delta},
\end{gathered}
$$

the energy level equation of Equation (31) for the nonrelativistic case, can be written the following form:

$$
E_{n_{r} l}^{N R}=-\frac{1}{2 M}\left[\delta\left(n_{r}+l+1\right)+\frac{M\left(V_{0}+2 \delta A\right)}{2 \delta\left(n_{r}+l+1\right)}\right]^{2},
$$

which is good agreement with the result in Equation (28) (If $\mathrm{B}$ and $\mathrm{C}$ are considered zero as a special case) of Ref [31]. Generally, it is obviously seen from Equation. (31) that the bound states show more stability in the case of the linear combination of Hulthén and Yukawa potentials than Hulthén and Yukawa potential cases. Furthermore, the energy eigenvalues of the quantum states are considerably sensitive with depending potential parameters

\section{Conclusion}

To conclude, we admit that the SUSY QM method was presented to solve the KFG equation for the linear combination of Hulthén and Yukawa potentials. Hence, the energy eigenvalues and corresponding eigenfunctions of a mentioned quantum system were analytically obtained for arbitrary $l$ angular momentum and $n_{r}$ radial quantum numbers. Next, a closed form of the normalization constant of the wave functions was also found. Beyond that, it was also shown that the energy eigenvalues are considerably sensitive respecting quantum states. Finally, the results obtained within SUSY QM are in excellent agreement with the results obtained by ordinary quantum mechanics, and it confirms that the mathematical application of SUSY quantum mechanics is ideal for similar systems. 
It is worth mentioning that the main results of this paper are the explicit and closed form expressions for the energy eigenvalues and the normalized wave functions. The method presented in this study is systematic, and in many cases, one of the most definite works in this field. In particular, the linear combination of Hulthén and Yukawa potentials can be one of the essential exponential potentials, and it probably provides a promising avenue in many branches of physics, especially in hadronic and nuclear physics.

\section{Appendix}

\section{A. SUSYQM Method}

For $N=2$ in SUSYQM, it is possible to define two nilpotent operators, $Q$ and $Q^{\dagger}$. They satisfy the following anticommutation relations:

$$
\begin{gathered}
\{Q, Q\}=0,\left\{Q^{\dagger}, Q^{\dagger}\right\}=0 \\
\left\{Q, Q^{\dagger}\right\}=H
\end{gathered}
$$

Here, $H$ is the supersymmetric Hamiltonian operator and conventionally $Q=\left(\begin{array}{cc}0 & 0 \\ A^{-} & 0\end{array}\right)$ and $Q^{\dagger}=\left(\begin{array}{cc}0 & A^{+} \\ 0 & 0\end{array}\right)$. The $Q$ and $Q^{\dagger}$ are also known as the supercharges operators. Here, $A^{-}$is bosonic operator and $A^{+}$is its adjoint. In terms of these operators, the Hamiltonian $H$ can be defined as $[27,28]$ :

$$
H=\left(\begin{array}{cc}
A^{+} A^{-} & 0 \\
0 & A^{-} A^{+}
\end{array}\right)=\left(\begin{array}{cc}
H_{-} & 0 \\
0 & H_{+}
\end{array}\right),
$$

where the $H_{+}$is named as the Hamiltonian of supersymmetric partner. Note also that $Q$ and $Q^{\dagger}$ operators commute with $H$. If we have zero ground state energy for $H$ (i.e., $E_{0}=0$ ), we can always represent the Hamiltonian as a product of a linear differential operators pairs in a factorable form. Therefore, the ground state $\psi_{0}(x)$ obeys the Schrödinger equation as follows:

$$
H \psi_{o}(x)=-\frac{\hbar^{2}}{2 m} \frac{d^{2} \psi_{0}}{d x^{2}}+V(x) \psi_{0}(x)=0
$$

hence,

$$
V(x)=\frac{\hbar^{2}}{2 m} \frac{\psi_{0}^{\prime \prime}(x)}{\psi_{0}(x)}
$$

This result makes us possible to globally reconstruct the above potential from the information of its ground state wave function that contain zero nodes. Hence, factorizing of $H$ is quite easy by using the following ansatz $[27,28]$ :

$$
H_{-}=-\frac{\hbar^{2}}{2 m} \frac{d^{2}}{d x^{2}}+V(x)=A^{+} A^{-}
$$

where

$$
A^{-}=\frac{\hbar}{\sqrt{2 m}} \frac{d}{d x}+W(x), A^{+}=-\frac{\hbar}{\sqrt{2 m}} \frac{d}{d x}+W(x) .
$$

After that, the Riccati equation for $W(x)$ can be written as

$$
V_{-}(x)=W^{2}(x)-\frac{\hbar}{\sqrt{2 m}} W^{\prime}(x)
$$

Solving for $W(x)$ from this equation, we can express it in terms of $\psi_{0}(x)$ by

$$
W(x)=-\frac{\hbar}{\sqrt{2 m}} \frac{\psi_{0}^{\prime}(x)}{\psi_{0}(x)}
$$

We obtain this solution by noticing that when $A^{-} \psi_{0}(x)$ $=0$ is satisfied, we have $H \psi_{0}=A^{+} A^{-} \psi_{0}=0$. We then introduce the operator $H_{+}=A^{-} A^{+}$which is written by reversing the order of the $H^{-}$components. After a bit simplification, we find that $H_{+}$is nothing but the Hamiltonian for new potential $V_{+}(x)$.

$$
H_{+}=-\frac{\hbar^{2}}{2 m} \frac{d^{2}}{d x^{2}}+V_{+}(x), V_{+}(x)=W^{2}(x)+\frac{\hbar}{\sqrt{2 m}} W^{\prime}(x) .
$$

We call $V_{+}(x)$ as supersymmetric partner potentials. For example, when the ground state energy of $H_{1}$ is $E_{0}^{1}$ with eigenfunction $\psi_{0}^{1}$, from Equation (A.5), we can always write

$$
H_{1}=-\frac{\hbar^{2}}{2 m} \frac{d^{2}}{d x^{2}}+V_{1}(x)=A^{+} A^{-}+E_{0}^{1},
$$

where

$$
\begin{gathered}
A_{1}^{-}=\frac{\hbar}{\sqrt{2 m}} \frac{d}{d x}+W_{1}(x), A_{1}^{+}=-\frac{\hbar}{\sqrt{2 m}} \frac{d}{d x}+W_{1}(x), \\
V_{1}(x)=W_{1}^{2}(x)-\frac{\hbar}{\sqrt{2 m}} W_{1}^{\prime}(x)+E_{0}^{1}, W_{1}(x)=-\frac{\hbar}{\sqrt{2 m}} \frac{d \ln \psi_{0}^{1}}{d x} .
\end{gathered}
$$

The SUSY partner Hamiltonian is defined by [27, 28].

$$
H_{2}=A_{1}^{-} A_{1}^{+}+E_{0}^{1}=-\frac{\hbar^{2}}{2 m} \frac{d^{2}}{d x^{2}}+V_{2}(x),
$$

where

$$
\begin{gathered}
V_{2}(x)=W_{1}^{2}(x)+\frac{\hbar}{\sqrt{2 m}} W_{1}^{\prime}(x)+E_{0}^{1} \\
=V_{1}(x)+\frac{2 \hbar}{\sqrt{2 m}} W_{1}^{\prime}(x)=V_{1}(x)-\frac{\hbar^{2}}{m} \frac{d^{2}}{d x^{2}}\left(\ln \psi_{0}^{(1)}\right) .
\end{gathered}
$$


Using Equation (A.12), for $H_{1}$ and $H_{2}$, the energy eigenvalues and eigenfunctions are obtained as

$E_{n}^{2}=E_{n+1}^{1}, \psi_{n}^{2}=\left[E_{n+1}^{1}-E_{0}^{1}\right]^{-1 / 2} A_{1}^{-} \psi_{n+1}^{1}, \psi_{n+1}^{1}=\left[E_{n}^{2}-E_{0}^{1}\right]^{-1 / 2} A_{1}^{+} \psi_{n}^{2}$.

Here $E_{n}^{m}$ represents the energy eigenvalue, where $n$ and $m$ denote the energy level and the $m$ th Hamiltonian $H_{m}$, respectively. Hence, it is clear that if $H_{1}$ has $p \geq 1$ bound states with corresponding eigenvalues $E_{n}^{1}$, as well as eigenfunctions $\psi_{n}^{1}$ defined in $0<n<p$, then we can always generate a hierarchy of $(p-1)$ Hamiltonians, i.e., $H_{2}, H_{3}, \cdots, H_{p}$ such that the $\left(H_{m}\right)$ has the same spectrum of eigenvalue as $H_{1}$, apart from the fact that the first $(m-1)$ eigenvalues of $H$ are absent in $H[27,28]$ :

$$
H_{m}=A_{m}^{+} A_{m}^{-}+E_{\mathrm{m}-1}^{1}=-\frac{\hbar^{2}}{2 m} \frac{d^{2}}{d x^{2}}+V_{m}(x)
$$

where

$$
A_{m}^{-}=\frac{\hbar}{\sqrt{2 m}} \frac{d}{d x}+W_{m}(x), \quad W_{m}(x)=-\frac{\hbar}{\sqrt{2 m}} \frac{d \ln \psi_{0}^{(m)}}{d x},(m=234, \cdots p)
$$

We also have

$$
\begin{gathered}
E_{n}^{(m)}=E_{n+1}^{(m-1)}=\cdots=E_{n+m-1}^{1}, \\
\psi_{n}^{(m)}=\left[E_{n+m-1}^{1}-E_{m-2}^{1}\right]^{-1 / 2} \cdots\left[E_{n+m-1}^{1}-E_{0}^{1}\right]^{-1 / 2} A_{m-1}^{-} \cdots A_{1}^{-} \psi_{n+m-1}^{1}, \\
V_{m}(x)=V_{1}(x)-\frac{\hbar^{2}}{m} \frac{d^{2}}{d x^{2}} \ln \left(\psi_{0}^{(1)} \cdots \psi_{0}^{(m-1)}\right) .
\end{gathered}
$$

such that, by knowing all the eigenfunctions and eigenvalues of $H_{1}$ we also obtain the corresponding eigenfunctions $\psi_{n}^{1}$ and energy eigenvalues $E_{n}^{1}$ of the $(p-1)$ Hamiltonians $\left(H_{2}\right.$, $\left.H_{3}, \cdots, H_{p}\right)$.

\section{Data Availability}

Data availability statement does not exist.

\section{Conflicts of Interest}

All authors have no conflicts of interest.

\section{References}

[1] S. L. Garavelli and F. A. Oliveira, "Analytical solution for a Yukawa-type potential," Physical Review Letters, vol. 66, no. 10, pp. 1310-1313, 1991.

[2] L. Boivin, F. X. Kartner, and H. A. Haus, "Analytical solution to the quantum field theory of self-phase modulation with a finite response time," Physical Review Letters, vol. 73, no. 2, pp. 240-243, 1994.
[3] I. B. Birula, "Particle beams guided by electromagnetic vortices: new solutions of the Lorentz, Schrödinger, Klein-Gordon, and Dirac equations," Physical Review Letters, vol. 93, no. 2, 2004.

[4] M. Belic, N. Petrovic, W. P. Zhong, R. H. Xie, and G. Chen, "Analytical light bullet solutions to the generalized $(3+1)$ dimensional nonlinear Schrödinger equation," Physical Review Letters, vol. 101, no. 12, 2008.

[5] W. Greiner, Relativistics Quantum Mechanics, Springer, Berlin, 3rd edition, 2000.

[6] V. G. Bagrov and D. M. Gitman, Exact Solutions of Relativistic Wave Equations, Kluwer Academic Publishers, Dordrecht; Boston, 1990.

[7] H. Feshbach and F. Villars, "Elementary relativistic wave mechanics of spin 0 and spin 1/2 particles," Reviews of Modern Physics, vol. 30, no. 1, pp. 24-45, 1958.

[8] A. F. Nikiforov and V. B. Uvarov, Special Functions of Mathematical Physics, Springer, Birkhäuser, Boston, 1988.

[9] S.-H. Dong, Factorization Method in Quantum Mechanics. Part of the Fundamental Theories of Physics Book Series, Springer, Dordrecht, 2007.

[10] A. Arda and R. Sever, "Exact solutions of the Morse-like potential, step-up and step-down operators via Laplace transform approach," Communications in Theoretical Physics, vol. 58, no. 1, pp. 27-30, 2012.

[11] J. M. Cai, P. Y. Cai, and A. Inomata, "Path-integral treatment of the Hulthén potential," Physical Review A, vol. 34, no. 6, pp. 4621-4628, 1986.

[12] A. Z. Tang and F. T. Chan, "Shifted1/Nexpansion for the Hulthén potential," Physical Review A, vol. 35, no. 2, pp. 911-914, 1987.

[13] B. Roy and R. Roychoudhury, "The shifted 1/N expansion and the energy eigenvalues of the Hulthen potential for 1 not $=0$," Journal of Physics A: Mathematical and General, vol. 20, no. 10, pp. 3051-3055, 1987.

[14] M. Hamzavi, A. A. Rajabi, and H. Hassanabadi, "Exact pseudospin symmetry solution of the Dirac equation for spatiallydependent mass Coulomb potential including a Coulomblike tensor interaction via asymptotic iteration method," Physics Letters A, vol. 374, no. 42, pp. 4303-4307, 2010.

[15] R. R. Hartmann and M. E. Portnoi, "Two-dimensional Dirac particles in a Pöschl-Teller waveguide," Scientific Reports, vol. 7, no. 1, p. 11599, 2017.

[16] B. C. Lütfüoğlu, J. Lipovský, and J. Kříž, "Scattering of KleinGordon particles in the background of mixed scalar-vector generalized symmetric Woods-Saxon potential," The European Physical Journal Plus, vol. 133, no. 1, 2018.

[17] B. C. Lütfüoğlu, "An investigation of the bound-state solutions of the Klein-Gordon equation for the generalized WoodsSaxon potential in spin symmetry and pseudo-spin symmetry limits," The European Physical Journal Plus, vol. 133, no. 8, 2018.

[18] V. H. Badalov, H. I. Ahmadov, and S. V. Badalov, "Any l-state analytical solutions of the Klein-Gordon equation for the Woods-Saxon potential," International Journal of Modern Physics E, vol. 19, no. 7, pp. 1463-1475, 2012.

[19] R. H. Parmar, "Construction of solvable non-central potential using vector superpotential: a new approach," Indian Journal of Physics, vol. 93, no. 9, pp. 1163-1170, 2019.

[20] J. Gao and M.-C. Zhang, "Pseudospin symmetric solution of the Dirac-Eckart problem with a Hulthén tensor interaction 
in the tridiagonal representation," Physics Letters B, vol. 769, pp. 77-81, 2017.

[21] P. Zhang, H. C. Long, and C. S. Jia, "Solutions of the Dirac equation with the Morse potential energy model in higher spatial dimensions," The European Physical Journal Plus, vol. 131, no. 4, 2016.

[22] M. Znojil, "Exact solution of the Schrodinger and KleinGordon equations for generalised Hulthen potentials," Journal of Physics A: Mathematical and General, vol. 14, no. 2, pp. 383394, 1981.

[23] F. Domínguez-Adame, "Bound states of the Klein-Gordon equation with vector and scalar Hulthen-type potentials," Physics Letters A, vol. 136, no. 4-5, pp. 175-177, 1989.

[24] G. Chen, Z.-D. Chen, and Z.-M. Lou, "Exact bound state solutions of the s-wave Klein-Gordon equation with the generalized Hulthen potential," Physics Letters A, vol. 331, no. 6, pp. 374-377, 2004.

[25] B. Talukdar, A. Yunus, and M. R. Amin, "Continuum states of the Klein-Gordon equation for vector and scalar interactions," Physics Letters A, vol. 141, no. 7, pp. 326-330, 1989.

[26] L. Chetouani, L. Guechi, A. Lecheheb, T. F. Hammann, and A. Messouber, "Path integral for Klein-Gordon particle in vector plus scalar Hulthen-type potentials," Physica A: Statistical Mechanics and its Applications, vol. 234, no. 1-2, pp. 529544, 1996.

[27] F. Cooper, A. Khare, and U. Sukhatme, Supersymmetry in Quantum Mechanics, World Scientific, 2001.

[28] F. Cooper, A. Khare, and U. Sukhatme, "Supersymmetry and quantum mechanics," Physics Reports, vol. 251, no. 5-6, pp. 267-385, 1995.

[29] D. A. Morales, "Supersymmetric improvement of the pekeris approximation for the rotating morse potential," Chemical Physics Letters, vol. 394, no. 1-3, pp. 68-75, 2004.

[30] S.-H. Dong, G.-H. Sun, and M. Lozada-Cassoua, "Exact solutions and ladder operators for a new anharmonic oscillator," Physics Letters A, vol. 340, no. 1-4, pp. 94-103, 2005.

[31] I. B. Okon, O. Popoola, and C. N. Isonguyo, "Approximate solutions of Schrodinger equation with some diatomic molecular interactions using Nikiforov-Uvarov method," Advances in High Energy Physics, vol. 2017, Article ID 9671816, 24 pages, 2017.

[32] M. Simsek and H. Egrifes, "The Klein-Gordon equation of generalized Hulthén potential in complex quantum mechanics," Journal of Physics A: Mathematical and General, vol. 37, no. 15, pp. 4379-4393, 2004.

[33] H. Egrifes and R. Sever, "Bound-state solutions of the KleinGordon equation for the generalized PT-symmetric Hulthén potential," International Journal of Theoretical Physics, vol. 46, no. 4, pp. 935-950, 2007.

[34] C.-Y. Chen, D.-S. Sun, and F.-L. Lu, “Approximate analytical solutions of Klein-Gordon equation with Hulthen potentials for nonzero angular momentum," Physics Letters A, vol. 370, no. 3-4, pp. 219-221, 2007.

[35] W.-C. Qiang, R.-S. Zhou, and Y. Gao, "Any $l$-state solutions of the Klein-Gordon equation with the generalized Hulthen potential," Physics Letters A, vol. 371, no. 3, pp. 201-204, 2007.

[36] S.-H. Dong and M. Lozada-Cassou, "Exact solutions of the Klein-Gordon equation with scalar and vector ring-shaped potentials," Physica Scripta, vol. 74, no. 2, pp. 285-287, 2006.

[37] G.-F. Wei, Z.-Z. Zhen, and S.-H. Dong, “The relativistic bound and scattering states of the Manning-Rosen potential with an improved new approximate scheme to the centrifugal term," Open Physics, vol. 7, no. 1, pp. 175-183, 2009.

[38] N. Saad, "The Klein-Gordon equation with a generalized Hulthén potential inD-dimensions," Physica Scripta, vol. 76, no. 6, pp. 623-627, 2007.

[39] F. Yasuk, A. Durmus, and I. Boztosun, "Exact analytical solution to the relativistic Klein-Gordon equation with noncentral equal scalar and vector potentials," Journal of Mathematical Physics, vol. 47, no. 8, p. 082302, 2006.

[40] L. Hulthén, “Arkiv foer Matematik," Astronomi, och Fysik, vol. 26, p. 5, 1942, 29B, 1.

[41] H. Yukawa, "On the Interaction of Elementary Particles. I," Proceedings of the Physico-Mathematical Society of Japan. $3 r d$ Series, vol. 17, pp. 48-57, 1935.

[42] H. I. Ahmadov, S. I. Jafarzade, and M. V. Qocayeva, "Analytical solutions of the Schrödinger equation for the Hulthén potential within SUSY quantum mechanics," International Journal of Modern Physics A, vol. 30, no. 32, article 1550193, 2015.

[43] V. H. Badalov, "The bound state solutions of the Ddimensional Schrödinger equation for the Woods-Saxon potential," International Journal of Modern Physics E, vol. 25, no. 1, p. 1650002, 2016.

[44] V. H. Badalov, B. Baris, and K. Uzun, "Bound states of the Ddimensional Schrödinger equation for the generalized WoodsSaxon potential," Modern Physics Letters A, vol. 34, no. 14, article 1950107, 2019.

[45] H. I. Ahmadov, M. V. Qocayeva, and N. S. Huseynova, "The bound state solutions of the D-dimensional Schrödinger equation for the Hulthén potential within SUSY quantum mechanics," International Journal of Modern Physics E, vol. 26, no. 5, p. 1750028, 2017.

[46] A. I. Ahmadov, M. Naeem, M. V. Qocayeva, and V. A. Tarverdiyeva, "Analytical bound-state solutions of the Schrödinger equation for the Manning-Rosen plus Hulthén potential within SUSY quantum mechanics," International Journal of Modern Physics A, vol. 33, no. 3, p. 1850021, 2018.

[47] A. I. Ahmadov, S. M. Nagiyev, M. V. Qocayeva, K. Uzun, and V. A. Tarverdiyeva, "Bound state solution of the Klein-FockGordon equation with the Hulthén plus a ring-shaped-like potential within SUSY quantum mechanics," International Journal of Modern Physics A, vol. 33, no. 33, p. 1850203, 2018.

[48] A. I. Ahmadov, M. Naeem, M. V. Qocayeva, and V. A. Tarverdiyeva, "Analytical solutions of the Schrödinger equation for the Manning-Rosen plus Hulthén potential within SUSY quantum mechanics," Journal of Physics: Conference Series, vol. 965, p. 012001, 2018.

[49] A. I. Ahmadov, M. Demirci, S. M. Aslanova, and M. F. Mustamin, "Arbitrary $\ell$-state solutions of the Klein-Gordon equation with the Manning-Rosen plus a class of Yukawa potentials," Physics Letters A, vol. 384, no. 12, p. 126372, 2020.

[50] A. A. Khelashvili and T. P. Nadareishvili, "What is the boundary condition for the radial wave function of the Schrödinger equation?," American Journal of Physics, vol. 79, no. 6, pp. 668-671, 2011.

[51] A. Khelashvili and T. Nadareishvili, "Dirac's reduced radial equations and the problem of additional solutions," International Journal of Modern Physics E, vol. 26, no. 7, p. 1750043, 2017.

[52] P. Matthys and H. De Meyer, "Dynamical-group approach to the Hulthén potential," Physical Review A, vol. 38, no. 3, pp. 1168-1171, 1988. 
[53] N. Ullah, "Screened Coulomb potential problem in the momentum representation," Physical Review A, vol. 40, no. 12, pp. 6831-6833, 1989.

[54] Y. P. Varshni, "Comparison of methods for improving the $1 / \mathrm{N}$ expansion,” Physical Review A, vol. 40, no. 4, pp. 2180-2183, 1989.

[55] Y. P. Varshni, "Eigenenergies and oscillator strengths for the Hulthén potential," Physical Review A, vol. 41, no. 9, pp. 4682-4689, 1990.

[56] C. Stubbins, "Bound states of the Hulthén and Yukawa potentials," Physical Review A, vol. 48, no. 1, pp. 220-227, 1993.

[57] S. H. Patil, "Simple wavefunctions for Yukawa- and Hulthéntype potentials," Journal of Physics A: Mathematical and General, vol. 34, no. 14, pp. 3153-3167, 2001.

[58] A. I. Ahmadov, S. M. Aslanova, M. S. Orujova, S. V. Badalov, and S.-H. Dong, "Approximate bound state solutions of the Klein-Gordon equation with the linear combination of Hulthen and Yukawa potentials," Physics Letters A, vol. 383, no. 24, pp. 3010-3017, 2019.

[59] L. É. Gendenshteïn, "Derivation of exact spectra of the Schrodinger equation by means of supersymmetry," JETP Letters, vol. 38, pp. 356-359, 1983.

[60] L. É. Gendenshteïn and I. V. Krive, "Supersymmetry in quantum mechanics," Soviet Physics Uspekhi, vol. 28, no. 8, pp. 645666, 1985.

[61] W.-C. Qiang and S.-H. Dong, "Arbitraryl-state solutions of the rotating Morse potential through the exact quantization rule method," Physics Letters A, vol. 363, no. 3, pp. 169-176, 2007.

[62] G.-F. Wei and S.-H. Dong, "Approximately analytical solutions of the Manning-Rosen potential with the spin-orbit coupling term and spin symmetry," Physics Letters A, vol. 373, no. 1, pp. 49-53, 2008.

[63] W.-C. Qiang and S.-H. Dong, "The Manning-Rosen potential studied by a new approximate scheme to the centrifugal term," Physica Scripta, vol. 79, no. 4, p. 045004, 2009.

[64] C. S. Jia, T. Chen, and L. G. Cui, "Approximate analytical solutions of the Dirac equation with the generalized Pöschl-Teller potential including the pseudo-centrifugal term," Physics Letters A, vol. 373, no. 18-19, pp. 1621-1626, 2009.

[65] R. L. Greene and C. Aldrich, "Variational wave functions for a screened Coulomb potential," Physical Review A, vol. 14, no. 6, pp. 2363-2366, 1976.

[66] M. Abramowitz and I. A. Stegun, Handbook of Mathematical Functions: with Formulas, Graphs and Mathematical Tables, Washington D.C., Dover Publications, New York, 1965.

[67] Z. Wang, Z.-W. Long, C.-Y. Long, and L.-Z. Wang, "Analytical solutions of position-dependent mass Klein-Gordon equation for unequal scalar and vector Yukawa potentials," Indian Journal of Physics, vol. 89, no. 10, pp. 1059-1064, 2015. 